Applied Multivariate Research, Volume 13, No. 2, 2010, 113-128

\title{
THE EFFECTS OF ESTIMATOR CHOICE AND WEIGHTING STRATEGIES ON CONFIRMATORY FACTOR ANALYSIS WITH STRATIFIED SAMPLES
}

\author{
Bradley J. Brummel ${ }^{1}$ \\ The University of Tulsa \\ Fritz Drasgow \\ University of Illinois at Urbana-Champaign
}

\begin{abstract}
Survey researchers often design stratified sampling strategies to target specific subpopulations within the larger population. This stratification can influence the population parameter estimates from these samples because they are not simple random samples of the population. There are three typical estimation options that account for the effects of this stratification in latent variable models: unweighted maximum likelihood, weighted maximum likelihood, and pseudo-maximum likelihood estimation. This paper examines the effects of these procedures on parameter estimates, standard errors, and fit statistics in Lisrel 8.7 (Jöreskog \& Sörbom, 2004) and Mplus 3.0 (Muthén \& Muthén, 2004). Options using several estimation methods will be compared to pseudo-maximum likelihood estimation. Results indicated the choice of estimation technique does not have a substantial effect on confirmatory factor analysis parameter estimates in large samples. However, standard errors of those parameter estimates and RMSEA values for assessing of model fit can be substantially affected by estimation technique.
\end{abstract}

Keywords: stratified samples, pseudo-maximum likelihood, parameter estimation, weighting strategies, latent variable modeling

\section{INTRODUCTION}

Researchers often employ complex sampling methodologies when surveying large populations. These methodologies include stratified sampling, cluster sampling, oversampling specific subpopulations, and other designs that lead to unequal probabilities of selection within the population (Skinner, Holt, \& Smith, 1989). For example, the Defense Manpower Data Center stratified their survey sample on the basis of reserve component, reserve program, gender, paygrade group, racial/ethnic group membership, and activation status for the 2004 Workplace and Gender Relations Survey of Reserve Component Members

1 Correspondence and requests for reprints should be addressed to Bradley J. Brummel, Department of Psychology, Lorton Hall, The University of Tulsa, 800 S. Tucker Dr., Tulsa, OK, 74104 or email at bradley-brummel@utulsa.edu. 
(Kroeger, 2004). These methodologies are used to obtain more reliable parameter estimates of subpopulations within the survey population. Stratified sampling can also be used to ensure a more closely matched sample to the actual population by taking random samples of a proportional size to the population within each subpopulation. Subpopulations are often chosen based upon geographic area, age, or minority group status within the population of interest. When the sampling procedure does not result in a simple random sample of the population, the survey sample will violate a basic assumption of most inferential statistical analyses. This violation can lead to biased estimates of population parameters unless the analysis accounts for the survey design (Skinner, et al.). Corrections are possible when informative sampling weights are available; in this situation, the sample cases can be weighted to appropriately represent the population (Asparouhov, 2005).

Correcting the sample estimates of means and variations to overall population parameter estimates is a straightforward task when a researcher has informative sampling weights. This is done by weighting each case in the sample back to its frequency in the overall population and adjusting the parameter estimates by this frequency. The population mean can be estimated by the weighted mean

$$
\hat{\mu}=\frac{\sum_{i=1}^{n} w_{i} y_{i}}{\sum_{i=1}^{n} w_{i}},
$$

where $w_{i}$ is the weight for the $i^{\text {th }}$ observation and $y_{i}$ is the observed score for the $i^{\text {th }}$ observation (Stapleton, 2002). The sampling variance of the estimator can be calculated by

$$
\operatorname{vâr}(\hat{\mu})=\frac{\sum_{i=1}^{n} w_{i}\left(y_{i}-\hat{\mu}\right)^{2}}{\sum_{i=1}^{n} w_{i}\left(\sum_{i=1}^{n} w_{i}-1\right)},
$$

where $w_{i}$ is the weight for the $i^{\text {th }}$ observation and $y_{i}$ is the observed score for the $i^{\text {th }}$ observation (Stapleton).

Obtaining accurate population estimates of parameters and fit statistics for latent variable models parameters is not as straightforward. These estimates require appropriate weighting throughout the process of matrix operations required for estimates of the model parameters and fit statistics. This issue has gained research interest in the past few years resulting in options for incorporating informative sampling weights in latent variable modeling programs including Lisrel Version 8.7 and Mplus Version 3.

Researchers have typically used one of three main weighting and estimation techniques for estimating population parameters for latent variables from sample data with unequal probability of selection. These methods are unweighted maximum likelihood (UML), weighted maximum likelihood (WML), and, pseudo-maximum likelihood (PML) estimation techniques. There has also been research examining the best scheme for scaling the sampling weights for the WML estimation (Kaplan \& Ferguson, 1999, Stapleton, 2002). These procedures include using the raw sample weights, relative sample weights, or effective 
sample weights. Each of these estimation techniques has implications for estimation bias and the resulting fit statistics for the estimated models.

This paper will describe these methods for estimating models from complex sampling designs. Then, it will examine the resulting parameter estimates, standard errors, and fit statistics for each of these techniques in the context of confirmatory factor analysis (CFA) of a measure of organizational commitment in a stratified sample using the procedures available in Lisrel 8.7 and Mplus 3.0. Finally, we will make suggestions regarding the utility of incorporating the various methods in typical scale evaluation and development, considering sample size and the degree of stratification.

\section{Estimation methods for data from stratified samples}

The three typical estimation methods for incorporating the information provided by knowledge of the sampling design into the estimation of population parameters in latent variable models are UML, WML, and PML. These methods also apply to the more limited use of sampling weights in the estimation of CFA parameters from stratified samples. By examining the difference between these techniques it is possible to make an informed decision about whether it is valuable to use the more complicated methods in a specific application.

Unweighted maximum likelihood. The UML estimation technique is not actually a technique for using the sampling weights from a study design. It is the practice of ignoring the information provided by the sampling weights. The sample data are treated as if they were a simple random sample of the larger population. The population parameters are also estimated as if the sample accurately reflected the distribution of individuals within the population of interest (Asparouhov, 2005).

For the estimation of some parameters, such as the population mean, an unweighted analysis can provide grossly biased estimates. For example, suppose Subpopulation A contains $90 \%$ of the cases in a population and has a mean of $\mu_{\mathrm{A}}=10$ and Subpopulation B contains the remaining $10 \%$ of the population and has a mean of 0 . If a researcher draws half of his/her sample from each population, the sample mean will converge to

$$
.5(10)+.5(0)=5,
$$

rather than the true population mean

$$
.9(10)+.1(0)=9 .
$$

Thus it is clear that this method will provide inaccurate parameter estimates when one or more of the subpopulations differ substantially on certain levels of a measured variable or have different interrelationships between some of the variables or items. Using UML estimation methods also tend to downwardly bias the standard error estimates from stratified samples (Kaplan \& Ferguson, 1999).

UML estimation has to be used if information about the sampling design is unavailable or non-informative. If there are varying response rates by subgroup or missing demographic information, then there may be little or no information available to create accurate sampling weights. If the sample is quite large in proportion to the population of interest, then there may be little to be gained by using the sample weights in the latent variable model estimation even if the sample weights are informative. UML is the simplest way to handle a stratified sample in latent variable model estimation, and this method can be implemented with any latent variable modeling statistics package. 
Weighted maximum likelihood. The WML estimation technique uses the weighted correlation matrix to estimate model parameters. However, there are multiple schemes that can be used for computing the weighted correlation matrix. Three of these are raw sample weights (WML), relative sample weights (WRML), and effective sample weights (WEML) (Stapleton, 2002). Relative and effective sample weights are suggested to normalize the weighting scales (Potthoff, Woodbury, \& Manton, 1992). Raw sample weights are the weights that produce the population size when applied to the sample. When these weights are applied, the effective sample $\mathrm{N}$ equals the population $\mathrm{N}$. This method allows unbiased estimation of the population parameters but produces standard errors and confidence intervals that are too small (Asparouhov, 2005). This results in overly liberal statistical inferences when using the chi-square difference tests of model fit.

Relative and effective sample weights (Potthoff, et al., 1992) normalize the weights by scaling the raw sample weights. Relative weights are calculated by multiplying the raw weights by

$$
\frac{n}{\sum_{i=1}^{n} w_{i}}
$$

where $w_{i}$ is raw weight for the $i^{\text {th }}$ observation. These relative sample weights recreate the actual sample size when applied to the sample because the average weight is equal to 1 . Interestingly, relative sample weights have also been shown to produce downwardly biased estimates of sampling variance in simulation studies (Stapleton, 2002).

Effective weights are calculated by multiplying the raw weights by

$$
\frac{\sum_{i=1}^{n} w_{i}}{\sum_{i=1}^{n} w_{i}^{2}} .
$$

These effective sample weights are designed to recreate the sample size that would produce an equivalent amount of information had the sampling procedure been a simple random sample. Therefore, the $n$ for the effective sample weighting technique is always less than or equal to the actual sample and relative sampling technique $n$ (Stapleton, 2002). This weighting technique has been shown to produce approximately unbiased estimates of model parameters and their sampling variances in simulation studies (Stapleton).

Mplus can approximate the raw sample weighted WML technique by treating the sample weights as if they were integer frequency weights (Asparouhov, 2005). It can not approximate the other weighting options as most weights in these designs will be between zero and one and will lose large amounts of information if rounded to the nearest integer (Muthén, 2005). Lisrel version 8.7 can incorporate any of these options for sample weights into its latent variable estimating procedures. This can be done through either the weight cases option or the survey design feature in Prelis. However, these weighting schemes do not remove all bias in the estimates. To accurately estimate all of the relevant fit statistics, parameters, and standard errors, the PML estimation technique should be used (Pfefferman, et al., 1998; Asparouhov). 
Pseudo-maximum likelihood. The PML estimation technique maximizes the weighted loglikelihood function,

$$
\log (L)=\sum_{i} w_{i} \log \left(L_{i}\right),
$$

where $L_{i}$ is the likelihood of the $i^{\text {th }}$ observation. This method calculates the covariance matrix by

$$
\left((\log (L))^{\prime \prime}\right)^{-1}\left(\sum_{i} w_{i}^{2}\left(\left(\log \left(L_{i}\right)\right)^{\prime}\right)^{T}\left(\log \left(L_{i}\right)\right)^{\prime}\right)\left((\log (L))^{\prime \prime}\right)^{-1},
$$

where ' and " represent the first and second derivative, $T$ indicates the transpose of a matrix, and the sum is over all individuals in the sample. PML provides consistent estimates under all sampling strategies (Asparouhov, 2005). This estimation method provides consistent parameter estimates for stratified samples while maintaining the accuracy of chi-square tests for model fit (Pfefferman, et al., 1998, Skinner 1989). PML is currently available in the statistics package Mplus Version 3.0. PML has been found to provide accurate fit statistics, parameter estimates, and estimated standard errors regardless of the scheme for the sample weights (Pfefferman, et al.).

\section{Study design}

This study will examine the parameter estimates, standard errors, and fit statistics obtained from the UML, WML, and PML techniques for a CFA model of an organizational commitment scale (Gade, Tiggle, \& Schumm, 2003) included in the 2004 Workplace and Gender Relations Survey of Reserve Component Members using the options available in Mplus 3.0 and Lisrel 8.7. These estimation techniques will be applied to a large, stratified sample with informative sampling weights and to small subsets of that sample to examine the effects of sample size. The results from the UML and various WML strategies will be compared to the PML technique. These analyses will allow us to make suggestions regarding the utility of the various methods for typical scale evaluation and development, considering sample size for the degree of stratification in this sample.

\section{METHOD}

\section{Participants}

The 2004 Workplace and Gender Relations Survey of Reserve Component Members was distributed to a stratified random sample of 76,031 Reserve component members from March 19, 2004 to June 21, 2004 using paper and pencil and web-based formats (Riemer, 2004). This yielded a usable sample of 26,443 United States Armed Forces reservists (men, $n=$ $12,902,49 \%$, women, $n=13,541,51 \%$ ).

Data were weighted to reflect the Reserve component population as of March 2004. The final sample weights were calculated using a three-step process. First the base weights were calculated to reflect variable probabilities of selection. The stratification categories included Reserve component, Reserve program, gender, paygrade group, racial/ethnic group membership, and activation status (Kroeger, 2004). The second step adjusted the base weights for non-response. Finally, the non-response-adjusted weights were adjusted to the known 
population totals as of the start of data collection. Complete details of weighting and response rates are reported by Flores-Cervantes, Jones, and Wilson (2004).

\section{Measure}

Meyer and Allen's (1990) Organizational Commitment scale was modified for more efficient use within military samples (Table 1). This modification involved removing items assessing normative commitment, using a military referent, and phrasing all items positively. It includes 4 items that assess affective commitment and 4 items that assess continuance commitment (Gade, et al., 2003). The scales demonstrated adequate reliability (alpha $=0.91$ for affective commitment and 0.88 for continuance commitment) and fit as a two factor model of commitment in the 2004 Workplace and Gender Relations Survey of Reserve Component Members sample (Ormerod, et al., 2005). This measure was analyzed with two underlying factors (Figure 1). Scale items were constrained to load only on the theoretically appropriate factor.

\section{Table 1}

\section{Organizational Commitment scale (Gade, Tiggle, \& Schumm, 2003)}

\section{Affective Commitment (AC)}

$\left(\mathrm{x}_{1}\right) \quad$ I feel like "part of the family" in the military.

$\left(\mathrm{x}_{2}\right) \quad$ The military has a great deal of personal meaning for me.

$\left(x_{3}\right) \quad$ I feel a strong sense of belonging to the military.

$\left(\mathrm{x}_{4}\right) \quad$ I feel emotionally attached to the military.

\section{Continuance Commitment (CC)}

$\left(\mathrm{x}_{5}\right) \quad$ It would be too costly for me to leave the military in the near future.

$\left(\mathrm{x}_{6}\right) \quad$ I am afraid of what might happen if I quit the military.

$\left(\mathrm{x}_{7}\right)$ Too much in my life would be interrupted if I decided I wanted to leave the military.

$\left(\mathrm{x}_{8}\right) \quad$ One of the problems of leaving the military would be the lack of available alternatives.

Note: Items response scale was a five point scale from strongly agree to strongly disagree

\section{Analytic Strategies}

Two-factor CFAs of the 8 item measure of affective and continuance commitment were conducted using the UML, WML, and PML estimation methods in Mplus 3.0 and using the UML, WML, WRML, and WEML methods in Lisrel 8.7. The WRML and WEML methods could not be used in Mplus because the program requires integer weights to conduct WML estimation with the UML estimator (Muthén, 2005). The WML raw weights were rounded to the nearest integer in the Mplus WML analysis so that the results of using sampling weights as frequency rates could be investigated (Asparouhov, 2005). The UML estimation method is identical in both software programs and therefore will only be reported once in the rest of the paper.

Complete data on the commitment measure were available for $98.1 \%(25,947)$ of the sample. Due to the high percentage of participants without missing data, participants with any missing data on the measure were deleted from further analyses. This resulted in the removal of 496 participants $(1.9 \%)$. The analyses were conducted using this sample of participants with complete data for the measure and for 10 randomly selected subsets of 250 participants 
from the larger sample. The average of the results for the 10 smaller subsets has been reported.

The informative sample weights in the 25,947 person group ranged from 0.96 to 183.70 with a mean of 29.70 and a median of 13.68 (Figure 2). These weights resulted in an overall population size of 770,641 when applied to the full sample. The relative sample weights ranged from 0.03 to 6.20 with a mean of 1.00 and a median of 0.46 . The effective sample weights ranged from 0.01 to 2.85 with a mean of 0.46 and a median of 0.21 . In the 250 participant subsets, the informative sample weights were rescaled by a factor of 103.78 $(25,946 / 250)$ to correspond to weights needed to recreate the population. The relative and effective sample weights for each of the 250 subsets were calculated as described above.

Figure 1

Two factor CFA of Affective and Continuance Commitment measure

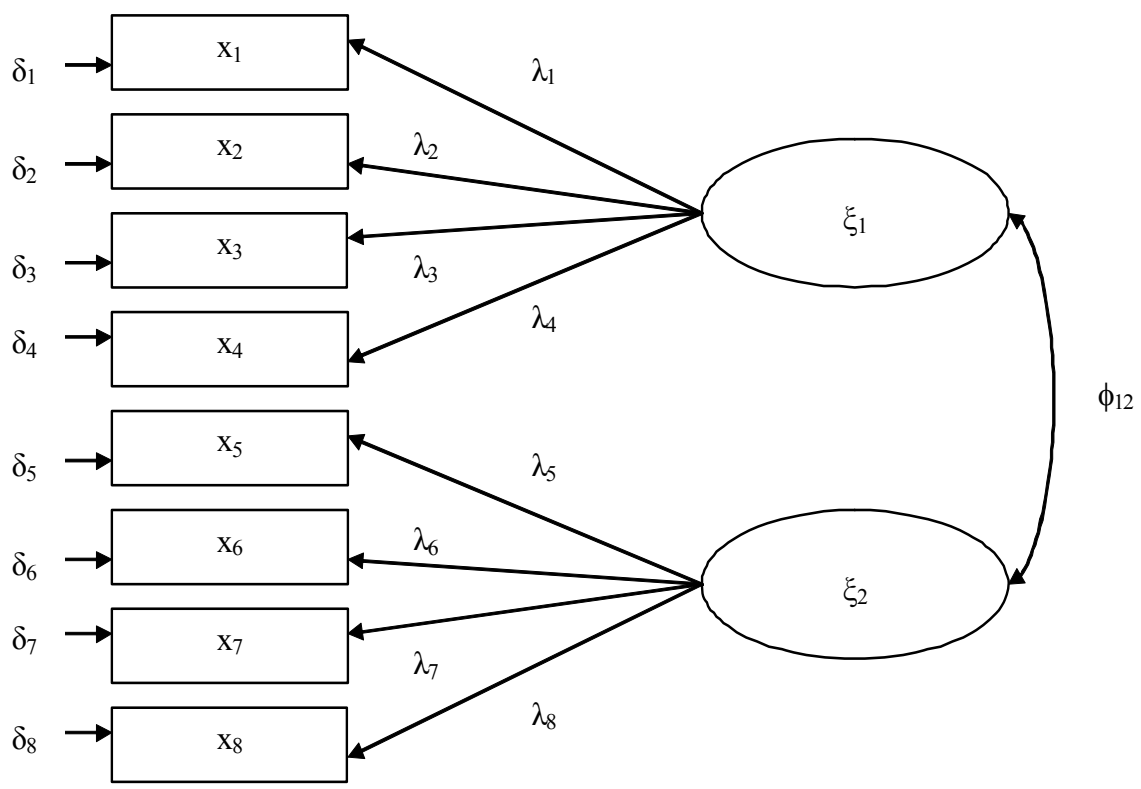

RESULTS

The estimates of means, variances, factor loadings, and factor correlation are given in Table 2. The largest differences in parameter estimates were observed for the item means and variances for the unweighted (UML) versus the weighted (WML, WRML, WEML, and PML) techniques. The weighted maximum likelihood estimation methods and the pseudo-maximum likelihood estimation method produced identical parameter estimates; however, the standard errors associated with these estimators were different. 
Figure 2

Distribution of raw sampling weights

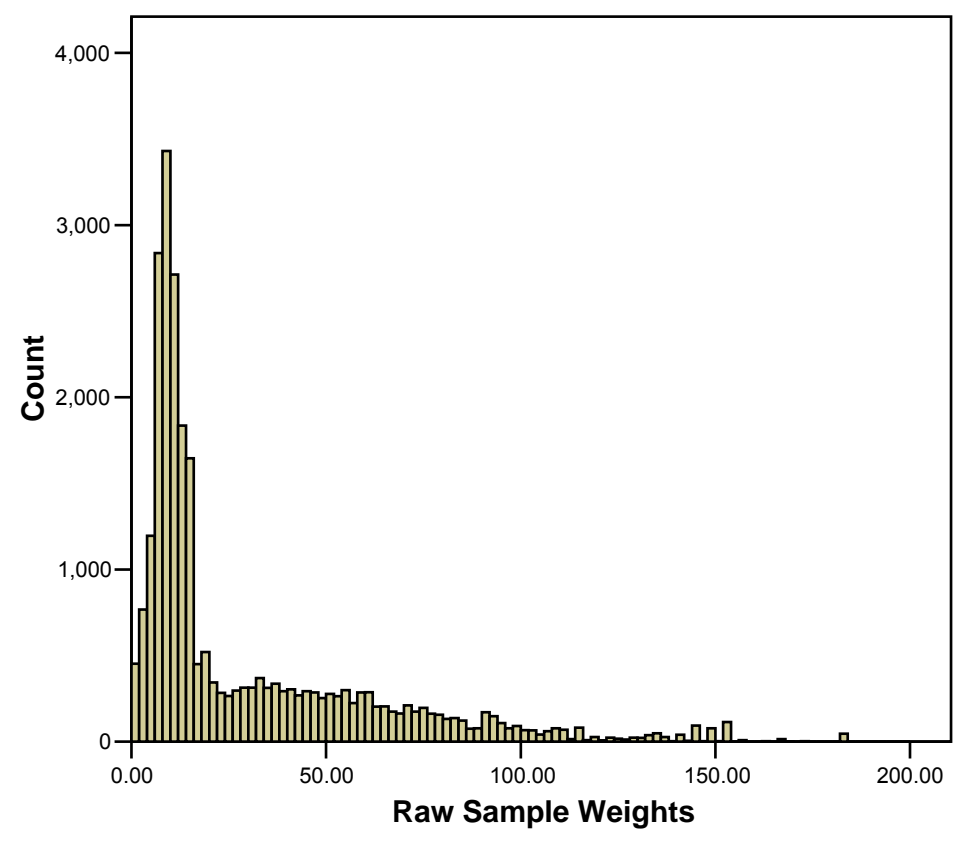

The use of the WML estimator in Mplus (WML(M)), which treats sample weights as frequency weights, resulted in much smaller standard error estimates than the PML estimator (Table 3); however, the use of WML techniques in Lisrel (WML(L)) resulted in standard error estimates identical to PML. This was also true for all weighting methods as the WML, WRML, and WEML methods all produced the same standard errors estimates. This occurs because Lisrel uses the actual sample size rather than the population size, relative sample size or effective sample size when estimating standard errors, so the weighting scheme does not affect these estimates. The UML standard errors were also smaller than the PML standard error estimates.

The resulting chi-square values, RMSEA, and effective sample sizes for the estimation techniques are presented in Table 4. The only fit statistics available from the WML methods in Lisrel were the full information UML Chi-square and the RMSEA because the program assumes that there is missing data when the weights are applied in Prelis. Also, the inclusion of sample weights in the PML technique does not allow chi-square difference tests to be used because the chi-square values are not distributed as chi-square (Muthén \& Muthén, 2004).

The WML(M) technique produced a much larger Chi-square value than the other techniques due to the inferred sample size. The UML Chi-square was also larger than the values for the other WML techniques and the PML technique. The WML weighting schemes again produced equivalent values in Lisrel, but not the same as the PML technique. 
Table 2

Parameter estimates for the full sample $(\mathrm{N}=\mathbf{2 5}, \mathbf{9 4 7})$ using $\mathrm{UML}, \mathrm{WML}$, and PML estimators

Unstandardized Standardized

\begin{tabular}{|c|c|c|c|c|c|c|}
\hline Statistic & UML & WML** & PML & UML & WML** & PML \\
\hline$\mu_{1}$ & 3.554 & 3.570 & 3.570 & & & \\
\hline$\mu_{2}$ & 3.628 & 3.612 & 3.612 & & & \\
\hline$\mu_{3}$ & 3.513 & 3.506 & 3.506 & & & \\
\hline$\mu_{4}$ & 3.237 & 3.205 & 3.205 & & & \\
\hline$\mu_{5}$ & 2.925 & 2.886 & 2.886 & & & \\
\hline$\mu_{6}$ & 2.578 & 2.551 & 2.551 & & & \\
\hline$\mu_{7}$ & 2.529 & 2.496 & 2.496 & & & \\
\hline$\mu_{8}$ & 2.641 & 2.624 & 2.624 & & & \\
\hline$\lambda_{1}$ & $1.000^{*}$ & $1.000^{*}$ & $1.000 *$ & .887 & .877 & .876 \\
\hline$\lambda_{2}$ & 1.055 & 1.024 & 1.024 & .892 & .897 & .897 \\
\hline$\lambda_{3}$ & 1.155 & 1.187 & 1.187 & 1.048 & 1.040 & 1.040 \\
\hline$\lambda_{4}$ & 1.059 & 1.127 & 1.127 & .996 & .988 & .988 \\
\hline$\lambda_{5}$ & $1.000^{*}$ & $1.000 *$ & $1.000^{*}$ & .918 & .940 & .940 \\
\hline$\lambda_{6}$ & 1.155 & 1,154 & 1,154 & 1.095 & 1.085 & 1.085 \\
\hline$\lambda_{7}$ & 1.187 & 1.188 & 1.188 & 1.120 & 1.117 & 1.117 \\
\hline$\lambda_{8}$ & .949 & .932 & .932 & .858 & .877 & .877 \\
\hline$\delta_{1}$ & .362 & .430 & .430 & & & \\
\hline$\delta_{2}$ & .290 & .320 & .320 & & & \\
\hline$\delta_{3}$ & .150 & .200 & .200 & & & \\
\hline$\delta_{4}$ & .285 & .415 & .415 & & & \\
\hline$\delta_{5}$ & .439 & .653 & .653 & & & \\
\hline$\delta_{6}$ & .251 & .403 & .403 & & & \\
\hline$\delta_{7}$ & .209 & .324 & .324 & & & \\
\hline$\delta_{8}$ & .495 & .694 & .694 & & & \\
\hline$\phi_{12}$ & .233 & .349 & .349 & .389 & .424 & .424 \\
\hline$\phi_{11}$ & .638 & .768 & .768 & 1.000 & 1.000 & 1.000 \\
\hline$\phi_{22}$ & .561 & .884 & .884 & 1.000 & 1.000 & 1.000 \\
\hline
\end{tabular}

Note: *Fixed value used for identification; **WRML and WEML methods

provided identical parameter estimates to WML

The various weighting strategies for the WML methods produced greatly different RMSEA values for the model. The UML and WML(M) RMSEA values were substantially larger than the PML value. It is important to note that these values would result in different conclusions about the fit of the model when using standard criteria such as RMSEA $<.05$ constituting reasonably good fit (Browne \& Cudeck, 1993): The UML, WML(M), and WEML solutions would be judged as providing poor fits, the WRML and the PML solutions would be evaluated as satisfactory, and the WML(L) solution might be viewed as aberrant because the uncommonly good fit of the model. It is important to note that there is disagreement as to the 
standards for model fit, especially for applied research. Whichever standards are chosen, the varying RMSEA values do create the possibility of reaching different conclusions regarding the acceptability of the model fit.

Table 3

Standard Error estimates for the full sample $(\mathrm{N}=25,947)$ using UML, WML, and PML estimators

\begin{tabular}{crrrr} 
Statistic & UML & WML(M)* & WML(L)** & PML \\
\hline$\mu_{1}$ & .007 & .001 & .010 & .010 \\
$\mu_{2}$ & .007 & .001 & .010 & .010 \\
$\mu_{3}$ & .007 & .001 & .010 & .010 \\
$\mu_{4}$ & .007 & .001 & .011 & .011 \\
$\mu_{5}$ & .008 & .001 & .011 & .011 \\
$\mu_{6}$ & .008 & .001 & .011 & .011 \\
$\mu_{7}$ & .008 & .001 & .011 & .011 \\
$\mu_{8}$ & .007 & .001 & .011 & .011 \\
\hline$\lambda_{1}$ & ----- & ----- & ----- & ------ \\
$\lambda_{2}$ & .007 & .001 & .010 & .010 \\
$\lambda_{3}$ & .007 & .001 & .011 & .011 \\
$\lambda_{4}$ & .007 & .001 & .012 & .012 \\
$\lambda_{5}$ & ------ & ------ & ----- & ------ \\
$\lambda_{6}$ & .008 & .001 & .012 & .012 \\
$\lambda_{7}$ & .008 & .001 & .012 & .012 \\
$\lambda_{8}$ & .008 & .001 & .012 & .012 \\
\hline$\delta_{1}$ & .004 & .001 & .009 & .009 \\
$\delta_{2}$ & .003 & .001 & .007 & .007 \\
$\delta_{3}$ & .005 & .001 & .006 & .006 \\
$\delta_{4}$ & .003 & .001 & .009 & .009 \\
$\delta_{5}$ & .003 & .001 & .013 & .013 \\
$\delta_{6}$ & .003 & .001 & .011 & .011 \\
$\delta_{7}$ & .003 & .001 & .011 & .011 \\
$\delta_{8}$ & .005 & .001 & .014 & .014 \\
\hline$\phi_{12}$ & .005 & .001 & .010 & .010 \\
$\phi_{11}$ & .008 & .002 & .016 & .016 \\
$\phi_{22}$ & .008 & .002 & .017 & .017 \\
\hline${ }_{2}$ & .001 &
\end{tabular}

Note: *Weighted maximum likelihood using frequency weights in Mplus; **Weighted maximum likelihood using design weights in Lisrel

The second set of analyses was conducted to evaluate the effects of sample size on the estimation techniques because large sample sizes often minimize differences between estimation techniques. Ten randomly selected sub-samples of 250 participants were taken from the full sample for these analyses. The various weighting strategies were recalculated in each of these sub-samples. Once the weighting strategies and the estimation techniques were 
applied to each of the 10 sub-samples, the results were averaged across the sub-samples. The results are reported in a similar manner to the first set in Tables 5, 6, and 7 .

Table 4

\begin{tabular}{|c|c|c|c|c|c|c|}
\hline \multicolumn{7}{|c|}{ Effective N, Chi-square, and RMSEA for the full sample $(\mathbf{N}=\mathbf{2 5 , 9 4 7 )}$} \\
\hline Statistic & UML & WML(M)* & WML(L)** & WRML & WEML & PML \\
\hline Effective $\mathrm{N}$ & 25,947 & 770,512 & 770,512 & 26,008 & 11,971 & 25,947 \\
\hline Chi-square & $4,003.9$ & $114,179.1$ & $1,291.5$ & $1,291.5$ & $1,291.5$ & $1,361.1$ \\
\hline RMSEA & .090 & .088 & .009 & .051 & .075 & .052 \\
\hline
\end{tabular}

As in the full sample, the parameter estimates were found to be very similar across the methods. The standard errors for WML(M) estimates are obviously much too small due to the assumed sample size based on the frequency weights. Interestingly, UML standard errors are considerably smaller than PML standard errors. Theoretically, the PML standard errors should be more accurate than the UML standard errors. However, further research is needed to verify this conjecture.

The Chi-square and RMSEA values (Table 7) also show a similar pattern of relative sizes to the results from the full sample analyses. However, the Chi-square value for the WML(M) analysis is much larger than in the full sample and the Chi-square values for the other methods are substantially smaller than in the full sample. The RMSEA values from the WML methods vary greatly and the UML and WML(M) values are larger than the PML value. Although the RMSEA was developed to be relatively unaffected by sample size, the conclusion that researchers might draw from Table 7 differ from those based on Table 4: Whereas PML and WRML appear satisfactory in Table 4, the RMSEA for the PML is $23 \%$ larger in Table 7. The WRML RMSEA results are similar in both analyses. Thus, a researcher using the PML technique might conclude that the 2-factor CFA model did not adequately fit in the smaller sample. Only the WML(L) and WRML RMSEAs would suggest that the CFA model fits well.

\section{DISCUSSION}

Our goal was to evaluate the effects of UML, WML, and PML estimation techniques on the CFA parameter estimates from large and small stratified samples with informative sampling weights. While the estimation techniques are not strictly comparable due to variations in their Mplus and Lisrel implementations and the assumptions of the techniques, we are able to examine the outcomes of using each technique. The different estimation techniques did not result in substantially different estimates of means, variances, factor loadings, and factor correlation in this sample of 25,947 participants with a range from 0.96 to 183.7 in the raw sample weights. Differences in the parameter estimates were larger in the sub-samples of 250 , but the differences were still not large. In general, as sample sizes decreases, the differences in the parameter estimates should on average increase.

The three estimation techniques did differ substantially in their estimates of standard errors of the parameter estimates. In the full sample, the UML and PML techniques resulted in similar standard error estimates; however, in the smaller stratified sample the UML method appears to estimate standard errors that are too small compared with the theoretically preferable PML method. This finding suggests caution when testing the significance of parameter estimates 
from small stratified samples when using UML estimation. The WML(M) technique estimates standard errors based on the assumption that the sampling weights are actually frequency weights. In order to enter the sample weights as frequency weights in Mplus, the sample weights had to be integer values, so the frequency weights that resulted were only approximations to the sample weights which had a range of 0.01 to 183.7. The frequency weights yielded an $\mathrm{N}$ of 770,641 instead of the correct $\mathrm{N}$ of 25,947 in the full sample and an average $\mathrm{N}$ of 758,538 instead of the correct $\mathrm{N}$ of 250 in the reduced samples. The resulting standard errors of WML(M) were dramatically smaller than they should be. Consequently, Type I error rates of significance tests of parameter estimates would be grossly inflated.

Table 5

Average parameter estimates for 10 reduced $(\mathrm{N}=250)$ samples using $\mathrm{UML}$, WML, and PML estimators

\begin{tabular}{|c|c|c|c|c|c|c|}
\hline \multirow[b]{2}{*}{ Statistic } & \multicolumn{3}{|c|}{ Unstandardized } & \multicolumn{3}{|c|}{ Standardized } \\
\hline & UML & WML** & PML & UML & WML** & PML \\
\hline$\mu_{1}$ & 3.566 & 3.635 & 3.635 & & & \\
\hline$\mu_{2}$ & 3.637 & 3.692 & 3.692 & & & \\
\hline$\mu_{3}$ & 3.500 & 3.580 & 3.580 & & & \\
\hline$\mu_{4}$ & 3.236 & 3.279 & 3.279 & & & \\
\hline$\mu_{5}$ & 2.984 & 3.010 & 3.010 & & & \\
\hline$\mu_{6}$ & 2.657 & 2.646 & 2.646 & & & \\
\hline$\mu_{7}$ & 2.594 & 2.599 & 2.599 & & & \\
\hline$\mu_{8}$ & 2.713 & 2.733 & 2.733 & & & \\
\hline$\lambda_{1}$ & $1.000^{*}$ & $1.000 *$ & $1.000 *$ & .896 & .883 & .883 \\
\hline$\lambda_{2}$ & 1.006 & .995 & .995 & .901 & .877 & .877 \\
\hline$\lambda_{3}$ & 1.155 & 1.135 & 1.135 & 1.035 & .996 & .996 \\
\hline$\lambda_{4}$ & 1.112 & 1.093 & 1.093 & .996 & .958 & .958 \\
\hline$\lambda_{5}$ & $1.000^{*}$ & $1.000 *$ & $1.000 *$ & .895 & .908 & .908 \\
\hline$\lambda_{6}$ & 1.189 & 1.166 & 1.166 & 1.064 & 1.057 & 1.057 \\
\hline$\lambda_{7}$ & 1.244 & 1.224 & 1.224 & 1.113 & 1.111 & 1.111 \\
\hline$\lambda_{8}$ & .955 & .911 & .911 & .853 & .826 & .826 \\
\hline$\delta_{1}$ & .438 & .361 & .361 & & & \\
\hline$\delta_{2}$ & .309 & .308 & .308 & & & \\
\hline$\delta_{3}$ & .218 & .223 & .223 & & & \\
\hline$\delta_{4}$ & .422 & .440 & .440 & & & \\
\hline$\delta_{5}$ & 699 & .661 & .661 & & & \\
\hline$\delta_{6}$ & .464 & .492 & .492 & & & \\
\hline$\delta_{7}$ & .333 & .327 & .327 & & & \\
\hline$\delta_{8}$ & .717 & .638 & .638 & & & \\
\hline$\phi_{12}$ & .316 & .320 & .320 & .393 & .409 & .399 \\
\hline$\phi_{11}$ & .804 & .788 & .788 & 1.000 & 1.000 & 1.000 \\
\hline$\phi_{22}$ & .802 & .827 & .827 & 1.000 & 1.000 & 1.000 \\
\hline
\end{tabular}

Note: *Fixed value used for identification; **WRML and WEML methods provided identical parameter estimates to WML 
Table 6

Average standard error estimates for 10 reduced samples

( $N=250)$ using UML, WML, and PML estimators

\begin{tabular}{|c|c|c|c|c|}
\hline Statistic & UML & WML(M)* & WML(L)** & PML \\
\hline$\mu_{1}$ & .070 & .001 & .098 & .098 \\
\hline$\mu_{2}$ & .067 & .001 & .096 & .096 \\
\hline$\mu_{3}$ & .072 & .001 & .100 & .100 \\
\hline$\mu_{4}$ & .075 & .001 & .107 & .107 \\
\hline$\mu_{5}$ & .078 & .001 & .112 & .112 \\
\hline$\mu_{6}$ & .080 & .002 & .117 & .117 \\
\hline$\mu_{7}$ & .079 & .001 & .115 & .115 \\
\hline$\mu_{8}$ & .076 & .001 & .103 & .103 \\
\hline$\lambda_{1}$ & ----- & ------ & ------ & ------ \\
\hline$\lambda_{2}$ & .065 & .001 & .085 & .085 \\
\hline$\lambda_{3}$ & .069 & .001 & .096 & .096 \\
\hline$\lambda_{4}$ & .074 & .001 & .107 & .107 \\
\hline$\lambda_{5}$ & ------ & ------ & ------ & ------ \\
\hline$\lambda_{6}$ & .093 & .002 & .124 & .124 \\
\hline$\lambda_{7}$ & .094 & .002 & .133 & .133 \\
\hline$\lambda_{8}$ & .089 & .001 & .122 & .122 \\
\hline$\delta_{1}$ & .047 & .001 & .121 & .121 \\
\hline$\delta_{2}$ & .036 & .001 & .078 & .078 \\
\hline$\delta_{3}$ & .034 & .001 & .060 & .060 \\
\hline$\delta_{4}$ & .048 & .001 & .086 & .086 \\
\hline$\delta_{5}$ & .073 & .001 & .119 & .119 \\
\hline$\delta_{6}$ & .061 & .001 & .110 & .110 \\
\hline$\delta_{7}$ & .056 & .001 & .100 & .100 \\
\hline$\delta_{8}$ & .070 & .001 & .108 & .108 \\
\hline$\phi_{12}$ & .064 & .001 & .099 & .099 \\
\hline$\phi_{11}$ & .107 & .002 & .147 & .147 \\
\hline$\phi_{22}$ & .124 & .002 & .167 & .167 \\
\hline $\begin{array}{l}\text { Note: *Wei } \\
\text { Mplus; **V } \\
\text { Lisrel }\end{array}$ & $\begin{array}{l}\text { aximun } \\
\text { maxin }\end{array}$ & $\begin{array}{l}\text { ikelihood usi } \\
\text { m likelihood }\end{array}$ & $\begin{array}{l}\text { frequency w } \\
\text { ing design we }\end{array}$ & \\
\hline
\end{tabular}

The WML(L) technique does not fall victim to the problem of treating sample weights as frequency weights; however, depending on the choice of weighting strategy, different RMSEA values were obtained and different interpretations concerning model fit might be made. It appears relative weighting scheme in Lisrel is the closest approximation to the PML results and appears to be the preferred weighting strategy when using Lisrel. 
Table 7

\begin{tabular}{lrrrrrr}
\multicolumn{8}{c}{$\begin{array}{c}\text { Average effective N, Chi-square, and RMSEA and for } 10 \text { reduced samples (N=250) } \\
\text { Statistic }\end{array}$} & UML & WML(M)* & WML(L)** & WRML & WEML & PML \\
\hline Effective N & 250 & 758,538 & 758,538 & 250 & 127 & 250 \\
Chi-square & 65.3 & $252,451.74$ & 32.56 & 32.6 & 32.6 & 40.7 \\
RMSEA & .094 & .129 & .001 & .048 & .070 & .064 \\
\hline Note: *Weighted maximum likelihood using frequency weights in Mplus; **Weighted \\
maximum likelihood using design weights in Lisrel
\end{tabular}

\section{CONCLUSION}

Our goal in this paper was to examine the utility of the various estimation techniques for typical scale evaluation and development from stratified samples with informative sampling weights. There are a few conclusions that appear warranted. First, in large samples it may be possible to simply ignore the sampling weights or use any scaling of the sample weights and estimation technique in CFA analyses without substantially affecting the parameters estimates. Even in small samples, these estimates are fairly robust. This is comforting given that informative sampling weights are not always available when analyzing large scale survey research datasets.

The results are not so comforting when the standard errors of those estimates are considered. The WML(M) technique, which misinterprets the sample weights as frequency weights, leads to severely underestimated standard errors for parameter estimates when the degree of stratification is moderate. This result depends upon the sampling design rather than the sample size. The UML standard errors, which are estimated when sampling weights are ignored, appear to be downwardly biased (Kaplan \& Ferguson, 1999). This suggests caution when making inferences from these estimates. The WML techniques available in Lisrel replicated the standard errors from the PML technique and therefore seem preferable; however, the RMSEA values for these techniques were not equivalent.

The RMSEA values from the WRML weighting scheme most closely approximated the PML results in the full sample, and the RMSEA values from the WEML weighting scheme most closely approximated the PML results in the full reduced sample. So, given the equivalence on the parameter estimates and standard errors for the other weighting schemes, either the relative or the effective weights may be appropriate when using Lisrel for estimating population parameters from stratified samples; however, the RMSEA values for the WRML were smaller than the PML values, which would lead to less conservation inferences about model fit. When evaluation of overall model fit is of great importance, it may be worth the time and expense to use the PML technique in Mplus Version 3.0.

In sum, the advantage of using the more complicated methods of CFA estimation will vary depending upon the sample size and the importance of the accuracy of the parameter estimates beyond the first decimal point. Moreover, it is almost always important to interpret parameter estimates in light of their standard errors and the extent to which the overall fit of the model is satisfactory. Consequently, our analyses suggest that the WRML, WEML, and PML methods provide the most trustworthy results for stratified random samples such as the 2004 Workplace Gender Relations survey. 


\section{REFERENCES}

Asparouhov, T. (2005). Sampling weights in latent variable modeling. Structural Equation Modeling, 12, 411-434.

Brown, M. W., \& Cudeck, R. (1993). Alternative ways of assessing model fit. In K. Bollen \& J. S. Long (Eds.), Testing Structural Equation Models (pp. 136-162). Newbury Park: Sage Publications

Flores-Cervantes, I., Jones, M. E., \& Wilson, M. J. (2004). Weighting for the 2004 Workplace and Gender Relations Survey of Reserve Component Members. In R. A. Riemer (Ed.), 2004 Workplace and Gender Relations Survey of Reserve Component Members: Statistical Methodology Report (Report No. 2004-019). Arlington, VA: DMDC

Gade, P. A. Tiggle, R. B; \& Schumm, W. R. (2003). The measurement and consequences of military organizational commitment in soldiers and spouses. Military Psychology, 15, 191-207.

Kaplan, D. \& Ferguson, A. J. (1999). On the utilization of sample weights in latent variable models. Structural Equation Modeling, 6, 305-321.

Kroeger, K. R. (2004). Sample design for the 2004 Workplace and Gender Relations Survey of reserve component members. In R. A. Riemer (Ed.), 2004 Workplace and Gender Relations Survey of Reserve Component Members: Statistical Methodology Report (Report No. 2004-019). Arlington, VA: DMDC.

Jöreskog, K.G. \& Sörbom, D. (2004). LISREL 8.7 for Windows [Computer Software]. Lincolnwood, IL: Scientific Software International, Inc.

Meyer, J.P., \& Allen, N.J. 1990. The measurement and antecedents of affective, continuance, and normative commitment to the organization. Journal of Occupational Psychology, 63, $1-18$.

Muthén, B. O. (1998-2004). Mplus technical appendices.. Los Angeles, CA: Muthén \& Muthén.

Muthén, L. K. (2005, March 2). Mplus discussion. Message posted to $\mathrm{http}: / /$ www.statmodel.com/discussion/messages/9/579

Muthén, L. K. and Muthén, B. O. (1998-2004). Mplus user's guide. Third Edition. Los Angeles, CA: Muthén \& Muthén.

Muthén, L. K. and Muthén, B. O. (2004). Mplus 3.0 [Computer Software]. Los Angeles, CA: Muthén \& Muthén.

Ormerod, A. J., Lawson, A. K., Lytell, M. C., Wright, C. V., Sims, C. S., Brummel, B.J., Drasgow, F., Lee, W. C., \& Fitzgerald, L. F. (2005). 2004 Workplace and Gender Relations Survey of Reserve Component Members: Scales and measures report (Report No. 2004-022). Arlington, VA: DMDC.

Pfeffermann, D., Skinner, C. J., Holmes, D. J., Goldstein H., \& Rasbash, J. (1998). Weighting for unequal selection probabilities in multilevel models. Journal of the Royal Statistical Society. Series B (Statistical Methodology), 60, 23-40.

Potthoff, R. F., Woodbury, M. A., \& Manton, K. G. (1992). "Equivalent sample size" and "equivalent degrees of freedom" refinements for inference using survey weights under superpopulation models. Journal of the American Statistical Association, 87, 383-396. 
Riemer, R. A. (Ed.). (2004). 2004 Workplace and Gender Relations Survey of Reserve Component Members: Statistical Methodology Report (Report No. 2004-019). Arlington, VA: DMDC.

Skinner, C. J. (1989). Domain means, regression, and multivariate analysis. In C. J. Skinner, D. Holt, and T. M. F. Smith (Eds.), Analysis of complex surveys (pp. 59-87). New York: Wiley.

Skinner, C. J., Holt, D., \& Smith, T. M. F. (1989). General Introduction. In C. J. Skinner, D. Holt, and T. M. F. Smith (Eds.), Analysis of complex surveys (pp. 5-20). New York: Wiley.

Stapleton, L. (2002). The incorporation of sample weights into multilevel structural equation models. Structural Equation Modeling, 9, 475-502. 